\title{
Determination of the 3-D Magnetic Vector Potential using Lorentz Transmission Electron Microscopy
}

\author{
C. Phatak ${ }^{1}$, E. Humphrey ${ }^{1}$, M. De Graef ${ }^{1}$, A.K. Petford-Long ${ }^{2}$ \\ ${ }^{1}$ Department of Materials Science and Engineering, Carnegie Mellon University, Pittsburgh PA 15213 \\ ${ }^{2}$ Argonne National Laboratory, 9700 S Cass Avenue, Argonne, IL 60439
}

The development of modern magnetic devices requires an understanding of not only the three dimensional (3-D) structure of the device but also a knowledge of the 3-D magnetic configuration in and around the device. Since the image formation process in transmission electron microscopy is inherently quantum-mechanical, an investigation of the magnetic interactions between nanoscale magnetic elements requires knowledge of the fundamental electromagnetic properties of the nanostructure, namely the electrostatic potential and the magnetic vector potential rather than the associated electric and magnetic fields. Most modern microscopy methods such as TEM, scanning electron microscopy and various probe microscopies usually result in two dimensional (2-D) projections of the true 3-D structure. In the present work, we present a method for the complete 3-D characterization of magnetic structures by means of the determination of the 3-D magnetic vector potential.

The total phase change, $e^{+} m$, of an electron wave traveling through a magnetic thin foil can be expressed in terms of tomographic quantities; the electrostatic phase shift, $e_{e}$, corresponds to the scalar x-ray transform of the electrostatic potential, whereas the magnetic phase shift, ${ }_{m}$, is described by the vector x-ray transform of the magnetic vector potential [1]. Using the Fresnel mode of Lorentz microscopy (LTEM) and the Transport-of-Intensity (TIE) formalism, the total phase shift can be recovered from a through-focus image series [2]. The electrostatic and magnetic phase shifts are separated by recording the total phase shifts for specimen tilt angles and + . Two tilt series about mutually perpendicular axes are needed to reconstruct two components of the magnetic vector potential; the third component is retrieved by imposing the Coulomb gauge, which can be written as: $\quad \mathbf{A}=0$. To verify our tomographic rconstruction algorithms, simulations were performed for Permalloy (Py) elements of various shapes with different magnetic configurations. Phase computations and TIE reconstructions were carried out, followed by tomographic reconstruction of the magnetic vector potential. The simulations were performed assuming $C_{s}=7 \mathrm{~m}$ in Lorentz mode (Tecnai F20) and a defocus of $5 \mathrm{~m}$. Fig. 1(a) shows the schematic representation of the magnetic vector potential (red) of a square particle with closure domain configuration (magnetic induction in blue), and (b) shows the basic setup for the vector field tomography in a TEM in the sample reference frame. All simulations showed good agreement between the theoretical and reconstructed vector potential.

Experiments were performed on bilayers of $\operatorname{Py}(27 \mathrm{~nm}) / \mathrm{Cr}(3 \mathrm{~nm})$, lithographically patterned into various shapes. LTEM was performed on a Tecnai F20 operating at $200 \mathrm{keV}$. Orthogonal tilt series were acquired over the range of $\left[\begin{array}{ll}70 & +70\end{array}\right]$ ( 5 increments). The sample was then flipped by 180 and two additional tilt series were acquired. At each tilt angle, a 3-image through-focus series was recorded. Fig. 2(a)-(c) shows representative through-focus series images of an ellipse-shaped particle with two vortices, and (d) shows the experimentally reconstructed 3D magnetic vector potential. We will report on phase reconstructions followed by a full tomographic reconstruction for this and other data sets. 


\section{References}

[1] C. Phatak, M. Beleggia, and M. De Graef, Ultramicroscopy, 108, 503 (2008).

[2] M. De Graef and Y. Zhu, eds., Magnetic Microscopy and its Applications to Magnetic Materials, Academic Press, 2000.

[3] This work was supported by the U.S Department of Energy, Basic Energy Sciences under contract number DE-FG02-01ER45893.

[4] The electron microscopy was carried out at the Electron Microscopy Center for Materials Research at Argonne National Laboratory, a U.S. Department of Energy Office of Science Laboratory operated under Contract No. DE-AC02-06CH11357 by UChicago Argonne, LLC.

(a)

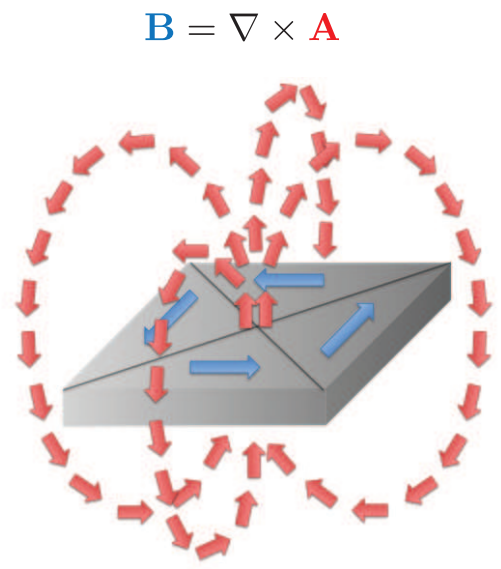

(b)

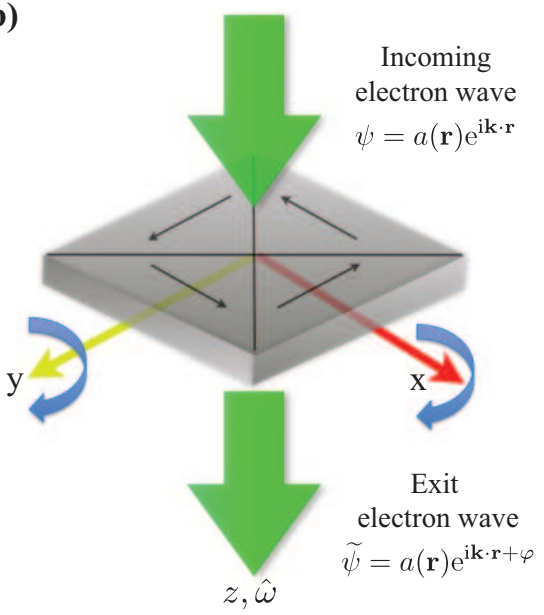

Figure 1: (a) Schematic representation of the 3D magnetic vector potential (red) of a square particle with closure domain configuration (magnetic induction in blue), and (b) shows the basic setup for the vector field tomography in a TEM in the sample reference frame.
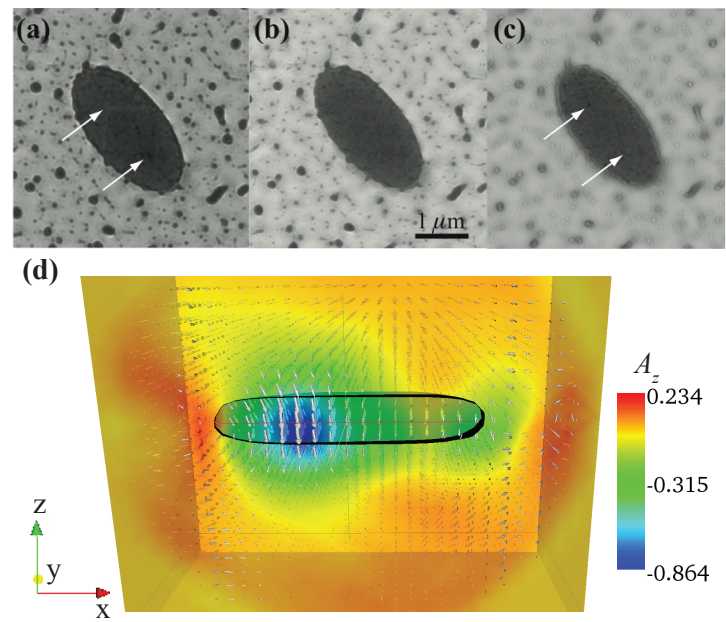

Figure 2: (a)-(c) Through Focus series of elliptical patricles of $\operatorname{Py}(27 \mathrm{~nm}) / \mathrm{Cr}(3 \mathrm{~nm})$, and (d) 3D Magnetic vector potential along the $x \quad z$ plane of the particle displayed as a vector field plot. The background is colored according the $z$ component of the vector potential. 